\title{
Análise da satisfação do paciente com o atendimento odontológico na Clínica de Odontologia da Universidade de Franca
}

\author{
Bruno Alves de Souza Toledo*, Alessandra Aparecida Campos**, Ronaldo
} Antônio Leite***

\author{
* Cirurgião-dentista, graduado pela Universidade de Franca \\ ** Professora Doutora. Docente responsável pela Disciplina de \\ Diagnóstico Integrado do Curso de Odontologia da Universidade \\ de Franca \\ *** Professor Doutor. Docente da Disciplina de Diagnóstico Integrado \\ do Curso de Odontologia da Universidade de Franca
}

\section{RESUMO}

Para a formação dos cirugiões-dentistas, é de grande importância o atendimento de pacientes durante o período de graduação, por isso, algumas Faculdades oferecem atendimento gratuito a pacientes sob a supervisão de um professor. Muitas avaliações são realizadas para verificar a qualidade do atendimento, no entanto, deixam de lado a visão dos pacientes que exercem um papel fundamental. Tendo em vista a importância do usuário para o processo de ensinoaprendizagem, o objetivo desse trabalho foi avaliar a satisfação dos pacientes com o serviço odontológico prestado pelas clínicas na Universidade de Franca, para assim poder analisar os aspectos do atendimento, e desenvolver novos métodos visando propiciar ao paciente um atendimento adequado e de qualidade. Foram entrevistados 81 pacientes, com o auxílio de um questionário proposto por Davies e Ware Jr. (1982) e adaptado para esta pesquisa, sendo compostas por 18 questões que permitiram avaliar o acesso, disponibilidade ou conveniência, custo, continuidade, satisfação geral, controle da dor, qualidade, acesso total e índice de satisfação com o atendimento odontológico, através de uma regra de escores. Diante dos resultados apresentados, encontrou-se que a maioria das questões apresentou mais de $70 \%$ de respostas positivas, no entanto, o item relativo à satisfação geral, que engloba apenas uma pergunta, onde o paciente diz se o atendimento poderia ser melhor, recebeu um percentual menor (56\%). Pode-se concluir que os pacientes apresentaram-se parcialmente satisfeitos com o atendimento realizados pelas clínicas da Universidade de Franca.

\section{DESCRITORES}

Avaliação de serviços. Faculdade de Odontologia. Satisfação.

$\mathbf{P}$ ara a formação de profissionais de saúde como os cirurgiões-dentistas, é de grande importância o atendimento de pacientes durante o período da graduação, assim os estudantes podem entrar no mercado de trabalho, capacitados a realizar procedimentos odontológicos, por isso, algumas faculdades de Odontologia oferecem atendimento gratuito a pacientes atendidos pelo sistema único de saúde (SUS).

Muitas vezes são realizadas avaliações para verificar a qualidade do atendimento prestado nas clínicas odontológicas das faculdades, mas estas avaliações, em sua maioria são realizadas por professores e alunos, deixando de lado a visão dos pacientes que exercem um papel fundamental no atendimento odontológico. ${ }^{1}$

O paciente atendido nas clínicas de odontologia das faculdades deve ser considerado um usuário dos serviços oferecidos, e não como um mero material de estudo como muitos pensam. ${ }^{7}$ Por isso os pacientes devem ser tratados com respeito e receber um tratamento de qualidade para que fiquem satisfeitos com o tratamento oferecido e tenham a certeza de que receberam o tratamento ideal.

Devido essa preocupação com a satisfação dos pa- 
cientes atendidos nas clínicas odontológicas de faculdades, pesquisas são realizadas para avaliar esse índice. Satisfação é definida como um processo complexo que equilibra expectativas do consumidor com percepções do serviço ou produto em questão ${ }^{9} \mathrm{e}$ uma resposta afetiva do paciente ajuda positiva ou negativamente nos efeitos atribuídos pela satisfação. ${ }^{11}$

São muitos os fatores que podem influenciar o nível satisfação do paciente, Lahti, Tuutti et al., ${ }^{6}$ (1995), avaliaram a opinião de vários pacientes e dentistas. Os pacientes mostraram que o dentista ideal é aquele profissional que concede informações e tem uma boa comunicação, e ainda destacaram a importância de uma aparência mais agradável. Já Brosky, Keefer et al. (2003), ${ }^{2}$ concluíram em seus estudos que os pacientes preferem que os estudantes de odontologia usem trajes mais formais, influenciando nos níveis de conforto e ansiedade, e acrescentaram ainda que o corte de cabelo, maquiagem e uso de jóias parece ter pouca influência, e destacaram que a primeira impressão que os pacientes têm dos alunos mostra um grande efeito sobre os níveis de confiança no atendimento.

Newsome e Wright, ${ }^{10}$ (1999)concluíram que a culpatibilidade, na definição dos pacientes "quando as coisas saem erradas", funciona como um filtro para a avaliação da satisfação dos pacientes, mostrado que se os pacientes tem uma experiência negativa com o serviço oferecido ele não saí satisfeito com o serviço oferecido.

A calma, paciência, capacidade de informação e comunicação são apontadas por paciente como características ideais de um cirurgião dentista. ${ }^{3}$ Alguns cirurgiões dentistas adotam métodos para a manutenção de pacientes nos consultórios odontológicos, como dar uma maior atenção no primeiro dia de consulta e passar as informações por meio de linguagem específica tendo como um método básico a orientação direta. ${ }^{5}$

Outro método de avaliação da satisfação com o atendimento odontológico foi demonstrado por Mascarenhas $(2001),{ }^{8}$ que avaliou a influência de dois modelos de tratamento adotados nas clínicas odontológicas de faculdade, o modelo tradicional onde o paciente faz um rodízio nas clínicas que necessita, e o modelo de cuidado completo, que são as clínicas integradas onde o paciente passa por todos os tratamentos necessitados em uma única clínica, como nos consultórios particulares. Verificaram que não há diferenças nesses casos.

A avaliação dos pacientes, sobre atendimento odontológico prestado nas faculdades permite uma melhor compreensão dos fatores negativos e positivos gerado pelo atendimento prestado, podendo assim levar a construção de idéias para uma melhoria do atendimento. Bottan, Sperb et al., ${ }^{1}$ (2006), observaram em uma pesquisa desenvolvida na Universidade do Vale do Itajaí, que os pacientes estavam insatisfeitos com o tempo de espera pelo atendimento, e com isso concluíram que seria necessária uma revisão na triagem dos pacientes, e sugeriram que os prontuários na consulta inicial fossem feitos antes do paciente entrar na clínica odontológica, e que poderiam ser realizados atendimentos durante o período de férias, obtendo assim, uma melhora no atendimento e respectivamente uma maior satisfação dos pacientes.

Entretanto a avaliação dos serviços odontológicos na Universidade de Franca nunca foi realizada, a não ser por professores e alunos, portanto houve a preocupação com a visão do paciente com o serviço que está sendo prestado nesta instituição. Sendo assim o objetivo desse trabalho foi avaliar a satisfação dos pacientes com o serviço odontológico prestado na Universidade de Franca, para assim analisar os aspectos positivos e negativos do atendimento, e desenvolver novos métodos de atendimento para propiciar ao paciente um atendimento mais adequado e de melhor qualidade.

\section{MATERIAIS E MÉTODOS}

Este trabalho recebeu aprovação do Comitê de Ética em Pesquisa da Universidade de Franca, através do protocolo número CAAE-0013.0.393.000-08. A pesquisa apresentou um caráter exploratório-descritivo. Uma amostra aleatória foi obtida com auxílio de testes estatísticos que nos permitiram uma avaliação que apresentasse significância, entre os pacientes que estavam agendados para o tratamento nas clínicas do Curso de Odontologia da Universidade de Franca.

Para que seja realizado o atendimento odontológico, ao chegar a clínica de odontologia da Unifran, os pacientes são encaminhados a uma sala de espera. A entrevista foi realizada por somente um aluno examinador, antes do paciente entrar para a sala de atendimento. Como critérios de inclusão foram definidos que os pacientes deveriam ter idade mínima de 18 anos, já estarem em tratamento na clínica de Odontologia por pelo menos três sessões, e que participassem da pesquisa por livre e espontânea vontade.

Foram entrevistados 81 indivíduos, de ambos os gêneros, sendo que $48(59,26 \%)$ pertenciam ao gênero feminino, sem distinção de raça. Para que fosse possível a realização das entrevistas, realizamos uma 
Tabela 1 - Índice de satisfação geral do paciente com o atendimento odontológico.

\begin{tabular}{|c|c|c|c|c|c|}
\hline Perguntas & $\begin{array}{l}\text { Concordo } \\
\text { totalmente }\end{array}$ & Concordo & $\begin{array}{l}\text { Não tenho } \\
\text { certeza }\end{array}$ & Discordo & $\begin{array}{l}\text { Discordo } \\
\text { totalmente }\end{array}$ \\
\hline \multicolumn{6}{|l|}{$\begin{array}{l}\text { 1. Os atendimentos nas Clínicas de odontologia da } \\
\text { UNIFRAN poderiam ser melhores }\end{array}$} \\
\hline \multicolumn{6}{|l|}{$\begin{array}{l}\text { 2. Os alunos de odontologia da UNIFRAN são } \\
\text { cuidadosos ao examinar seus pacientes }\end{array}$} \\
\hline \multicolumn{6}{|l|}{$\begin{array}{l}\text { 3. Você evita ir às clínicas de odontologia da } \\
\text { UNIFRAN porque é doloroso }\end{array}$} \\
\hline \multicolumn{6}{|l|}{$\begin{array}{l}\text { 4. O paciente espera muito tempo para ser atendido } \\
\text { pelos alunos de odontologia da UNIFRAN }\end{array}$} \\
\hline \multicolumn{6}{|l|}{$\begin{array}{l}\text { 5. Os alunos de odontologia da UNIFRAN sempre } \\
\text { tratam seus pacientes com respeito }\end{array}$} \\
\hline \multicolumn{6}{|l|}{$\begin{array}{l}\text { 6. Existe um número suficiente de alunos nas clínicas } \\
\text { de odontologia da UNIFRAN }\end{array}$} \\
\hline \multicolumn{6}{|l|}{$\begin{array}{l}\text { 7. Os estudantes necessitam fazer mais para reduzir a } \\
\text { dor dos pacientes }\end{array}$} \\
\hline \multicolumn{6}{|l|}{$\begin{array}{l}\text { 8. A clínica de odontologia da UNIFRAN é bem } \\
\text { localizada }\end{array}$} \\
\hline \multicolumn{6}{|l|}{$\begin{array}{l}\text { 9. Os estudantes de odontologia da UNIFRAN sempre } \\
\text { evitam despesas desnecessárias para o paciente }\end{array}$} \\
\hline \multicolumn{6}{|l|}{$\begin{array}{l}\text { 10. Os estudantes de odontologia da UNIFRAN não } \\
\text { têm conhecimento profundo como deveriam ter }\end{array}$} \\
\hline \multicolumn{6}{|l|}{$\begin{array}{l}\text { 11. Você é atendido sempre pelos mesmos alunos } \\
\text { quando necessita de cuidados }\end{array}$} \\
\hline \multicolumn{6}{|l|}{$\begin{array}{l}\text { 12. É difícil confirmar um horário de atendimento na } \\
\text { clínica de odontologia da UNIFRAN imediatamente }\end{array}$} \\
\hline \multicolumn{6}{|l|}{$\begin{array}{l}\text { 13. Os estudantes de odontologia da UNIFRAN } \\
\text { são capazes de aliviar ou curar a maioria dos } \\
\text { problemas dentários que as pessoas têm }\end{array}$} \\
\hline \multicolumn{6}{|l|}{$\begin{array}{l}\text { 14. Os horários de atendimento da clínica de } \\
\text { odontologia da UNIFRAN são bons para a maioria } \\
\text { dos pacientes }\end{array}$} \\
\hline \multicolumn{6}{|l|}{$\begin{array}{l}\text { 15. Os estudantes sempre explicam o que vão fazer, } \\
\text { antes de começar o tratamento }\end{array}$} \\
\hline \multicolumn{6}{|l|}{$\begin{array}{l}\text { 16. Os estudantes de odontologia da UNIFRAN devem } \\
\text { fazer mais para evitar que os pacientes tenham } \\
\text { problemas com seus dentes }\end{array}$} \\
\hline \multicolumn{6}{|l|}{$\begin{array}{l}\text { 17. As clínicas de odontologia da UNIFRAN são } \\
\text { modernas e atualizadas }\end{array}$} \\
\hline $\begin{array}{l}\text { 18. Eu não me preocupo em sentir dor quando recebo } \\
\text { cuidados odontológicos na clínica de odontologia } \\
\text { da UNIFRAN }\end{array}$ & & & & & \\
\hline
\end{tabular}

adaptação no questionário proposto por Davies e Ware Jr, $1982^{4}$ (Tabela 1). Essas alterações que foram realizadas não interferiram nos resultados.

Na entrevista, o entrevistador fazia uma afirmação e o paciente respondia se concordava totalmente, concordava, não tinha certeza, discordava, ou discordava totalmente. Para que fosse possível analisar estatisticamente as respostas, foram determinados escores de 1 a 5 sendo que quanto maior o escore maior a satisfação (Tabela 2).

Os resultados da soma dos escores de determinadas perguntas permitiam avaliar os aspectos em que os pacientes estavam ou não satisfeitos com o tratamento. Esses aspectos foram:

- acesso,

- disponibilidade/conveniência,

- não custo,

- continuidade, 
Tabela 2 - Escala para avaliação dos itens abordados.

\begin{tabular}{|l|c|}
\hline \multicolumn{1}{|c|}{ Escore } & Número do item \\
\hline 1 = Concordo totalmente & \\
\hline 2 = Concordo & $1,3,4,7$, \\
\hline $3=$ Não tenho certeza & $10,12,16$ \\
\hline $4=$ Discordo & \\
\hline $5=$ Discordo totalmente & \\
\hline $5=$ Discordo totalmente & $2,5,6,8,9,11$, \\
\hline $4=$ Discordo & $13,14,15,17,18$ \\
\hline $3=$ Não tenho certeza & \\
\hline $2=$ Concordo & \\
\hline $1=$ Concordo Totalmente & \\
\hline
\end{tabular}

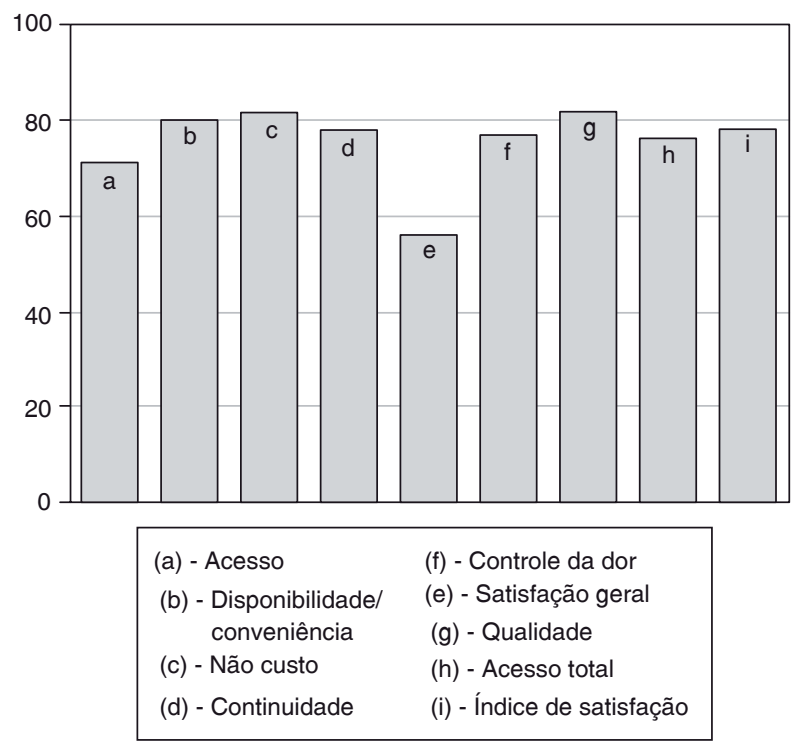

Gráfico 1 - Resultados dos escores de avaliação do atendimento indicado pelos usuários das clínicas do curso de odontologia.

- satisfação geral,

- controle da dor,

- qualidade,

- acesso total e

- índice de satisfação (Tabela 3).

\section{RESULTADOS}

Os dados obtidos foram expressos em gráficos para melhor compreensão e entendimento dos resultados. No Gráfico 1 encontra-se os resultados da avaliação do atendimento indicado pelos usuários das clínicas do curso de Odontologia da Universidade de Franca. Diante dos escores avaliados, a maioria dos itens apresentou mais de $70 \%$ de respostas positivas, o entanto o item relativo à satisfação geral, que en-
Tabela 3 - Escalas de variação para os escores.

\begin{tabular}{|l|l|}
\hline \multicolumn{1}{|c|}{ Escala } & Soma dos escores para esses itens \\
\hline $\begin{array}{l}\text { Acesso } \\
\text { Disponibilidade/ } \\
\text { conveniência }\end{array}$ & $4+12+14$ \\
\hline $\begin{array}{l}\text { Não custo } \\
\text { Continuidade }\end{array}$ & 9 \\
\hline $\begin{array}{l}\text { Satisfação geral } \\
\text { Controle da dor }\end{array}$ & 11 \\
\hline $\begin{array}{l}\text { Qualidade } \\
\text { Acesso total }\end{array}$ & $2+7+18$ \\
\hline $\begin{array}{l}\text { Índice de } \\
\text { satisfação }\end{array}$ & $4+6+8+9+12+14$ \\
\hline
\end{tabular}

globa apenas a pergunta 1 , recebeu um percentual menor que $63 \%$.

Como se pode observar na Tabela 4 estão apresentados os resultados expressos por percentual das respostas negativas ou positivas de todos os pacientes apresentados, vale ressaltar que as respostas que apresentam um maior escore (4 ou 5 ) indica uma maior satifação do paciente.

\section{DIscusSÃo}

Atualmente pesquisas apresentam a necessidade de se avaliar a satisfação dos pacientes com o atendimento odontológico, oferecido nas faculdades de Odontologia para que através dos resultados obtidos seja possível mudar aspectos negativos referentes ao tratamento gerando assim um melhor atendimento. ${ }^{1-3,8}$

Para avaliação da satisfação quanto maior o valor do escore, maior significa o grau da satisfação do paciente que recebeu os cuidados da clínica odontológica. Quando as perguntas foram realizadas separadamente geraram maior preocupação as que analisavam se o atendimento poderia ser melhor. $63 \%$ dos pacientes responderam que sim.

Na quarta pergunta que avaliava se o paciente esperava muito tempo na sala de espera para ser atendido, $57 \%$ dos pacientes responderam positivamente, indicando que se deve chamar esse paciente para o atendimento prontamente, ou seja, o paciente não deve esperar. Se necessário esperar, esse momento deve ser utilizado com palestras informativas, o que foi evidenciado com as respostas para a pergunta 16 que avaliou se os estudantes poderiam fazer mais para prevenir que os problemas bucais dos seus pacientes. $80 \%$ dos pacientes responderam que sim, ou seja, que deveria ser realizado debates, palestras, panfletagem 
Tabela 4 - Porcentagem da satisfação dos pacientes.

\begin{tabular}{|c|c|c|c|c|c|}
\hline Perguntas & $\begin{array}{l}\text { Concordo } \\
\text { totalmente }\end{array}$ & Concordo & $\begin{array}{l}\text { Não tenho } \\
\text { certeza }\end{array}$ & Discordo & $\begin{array}{l}\text { Discordo } \\
\text { totalmente }\end{array}$ \\
\hline $\begin{array}{l}\text { 1. Os atendimentos nas Clínicas de odontologia da } \\
\text { UNIFRAN poderiam ser melhores }\end{array}$ & $25 \%$ & $33 \%$ & $5 \%$ & $14 \%$ & $23 \%$ \\
\hline $\begin{array}{l}\text { 2. Os alunos de odontologia da UNIFRAN são } \\
\text { cuidadosos ao examinar seus pacientes }\end{array}$ & $85 \%$ & $14 \%$ & $0 \%$ & $0 \%$ & $1 \%$ \\
\hline $\begin{array}{l}\text { 3. Você evita ir às clínicas de odontologia da } \\
\text { UNIFRAN porque é doloroso }\end{array}$ & $2 \%$ & $6 \%$ & $0 \%$ & $25 \%$ & $67 \%$ \\
\hline $\begin{array}{l}\text { 4. O paciente espera muito tempo para ser atendido } \\
\text { pelos alunos de odontologia da UNIFRAN }\end{array}$ & $21 \%$ & $32 \%$ & $4 \%$ & $17 \%$ & $26 \%$ \\
\hline $\begin{array}{l}\text { 5. Os alunos de odontologia da UNIFRAN sempre } \\
\text { tratam seus pacientes com respeito }\end{array}$ & $80 \%$ & $19 \%$ & $1 \%$ & $0 \%$ & $0 \%$ \\
\hline $\begin{array}{l}\text { 6. Existe um número suficiente de alunos nas } \\
\text { clínicas de odontologia da UNIFRAN }\end{array}$ & $48 \%$ & $26 \%$ & $9 \%$ & $10 \%$ & $7 \%$ \\
\hline $\begin{array}{l}\text { 7. Os estudantes necessitam fazer mais para reduzir } \\
\text { a dor dos pacientes }\end{array}$ & $12 \%$ & $12 \%$ & $14 \%$ & $25 \%$ & $37 \%$ \\
\hline $\begin{array}{l}\text { 8. A clínica de odontologia da UNIFRAN é bem } \\
\text { localizada }\end{array}$ & $58 \%$ & $17 \%$ & $2 \%$ & $14 \%$ & $9 \%$ \\
\hline $\begin{array}{l}\text { 9. Os estudantes de odontologia da UNIFRAN } \\
\text { sempre evitam despesas desnecessárias para o } \\
\text { paciente }\end{array}$ & $51 \%$ & $26 \%$ & $7 \%$ & $9 \%$ & $7 \%$ \\
\hline $\begin{array}{l}\text { 10. Os estudantes de odontologia da UNIFRAN não } \\
\text { têm conhecimento profundo como deveriam ter }\end{array}$ & $14 \%$ & $19 \%$ & $5 \%$ & $23 \%$ & $40 \%$ \\
\hline $\begin{array}{l}\text { 11. Você é atendido sempre pelos mesmos alunos } \\
\text { quando necessita de cuidados }\end{array}$ & $46 \%$ & $26 \%$ & $9 \%$ & $11 \%$ & $9 \%$ \\
\hline $\begin{array}{l}\text { 12. É difícil confirmar um horário de atendimento } \\
\text { na clínica de odontologia da UNIFRAN } \\
\text { imediatamente }\end{array}$ & $15 \%$ & $16 \%$ & $7 \%$ & $19 \%$ & $43 \%$ \\
\hline $\begin{array}{l}\text { 13. Os estudantes de odontologia da UNIFRAN } \\
\text { são capazes de aliviar ou curar a maioria dos } \\
\text { problemas dentários que as pessoas têm }\end{array}$ & $59 \%$ & $30 \%$ & $7 \%$ & $2 \%$ & $1 \%$ \\
\hline $\begin{array}{l}\text { 14. Os horários de atendimento da clínica de } \\
\text { odontologia da UNIFRAN são bons para a maioria } \\
\text { dos pacientes }\end{array}$ & $57 \%$ & $22 \%$ & $0 \%$ & $14 \%$ & $7 \%$ \\
\hline $\begin{array}{l}\text { 15. Os estudantes sempre explicam o que vão fazer, } \\
\text { antes de começar o tratamento }\end{array}$ & $69 \%$ & $20 \%$ & $4 \%$ & $4 \%$ & $4 \%$ \\
\hline $\begin{array}{l}\text { 16. Os estudantes de odontologia da UNIFRAN } \\
\text { devem fazer mais para evitar que os pacientes } \\
\text { tenham problemas com seus dentes }\end{array}$ & $48 \%$ & $25 \%$ & $7 \%$ & $11 \%$ & $9 \%$ \\
\hline $\begin{array}{l}\text { 17. As clínicas de odontologia da UNIFRAN são } \\
\text { modernas e atualizadas }\end{array}$ & $74 \%$ & $23 \%$ & $2 \%$ & $0 \%$ & $0 \%$ \\
\hline $\begin{array}{l}\text { 18. Eu não me preocupo em sentir dor quando } \\
\text { recebo cuidados odontológicos na clínica de } \\
\text { odontologia da UNIFRAN }\end{array}$ & $40 \%$ & $25 \%$ & $0 \%$ & $10 \%$ & $26 \%$ \\
\hline
\end{tabular}

para a prevenção. Nas demais perguntas, os pacientes apresentaram-se satisfeitos, sendo que essa satisfação variou entre $62 \%$ na pergunta sete e $99 \%$ na pergunta dois.

Bottan, Sperb et al., ${ }^{1}$ (2006) relataram que a qualidade percebida pelo paciente está muito mais relacionada com a maneira que ele recebe o tratamento e com as pistas de qualidade que ele vai encontrar no consultório e no profissional do que com a parte técnica. Foi possível observar nos resultados que os pacientes apresentam uma boa impressão do serviço prestado e da arquitetura e modernidade do prédio onde estão instaladas as clínicas de Odontologia da Universidade de Franca, pois na questão que avaliava se a clínica estava bem localizada e se eram modernas e atualizadas, a grande maioria dos pacientes (97\%) 
estavam satisfeitos com o atendimento. Em serviço prestado, a maioria dos pacientes (89\%) concordaram que os estudantes estão capacitados a resolver a maioria dos problemas bucais dos pacientes.

Como resultados esta pesquisa apresentou mais de $70 \%$ na maioria das questões avaliadas, exceto no índice de satisfação geral que obteve um índice de $58 \%$, esta questão de satisfação geral englobava apenas a pergunta um, que avaliou se o atendimento poderia ser melhor, os pacientes relataram estar satisfeitos com o atendimento, porém acrescentaram que o atendimento poderia ser melhor, sugeriram que fossem realizados atendimentos noturnos e aos sábados.

Em conclusão Mascarenhas (2001), ${ }^{8}$ relatou que os pacientes avaliados estavam satisfeitos com o tratamento oferecido na Faculdade de Odontologia da Universidade, pois não apresentou diferenças significantes nas questões que permitiam avaliar o acesso, controle da dor e qualidade do serviço prestado, entretanto nesse estudo observaram que os pacientes estavam insatisfeitos com o tempo de espera na sala de espera, isto também foi visto nos resultados apresentados na pesquisa de Bottan et al., ${ }^{1}$ (2006). Quando analisamos as afirmações separadamente os pacientes apresentaram-se satisfeitos com o tempo de espera pelo tratamento nas clínicas do Curso de Odontologia da Universidade de Franca.

Analisando as afirmações separadamente, o item que analisava se os estudantes deveriam fazer mais para evitar que os pacientes tenham problemas com seus dentes, gerou muita polêmica, pois os pacientes sugeriram que poderiam ser realizadas mais campanhas e debates com os pacientes para incentivar hábitos que prevenissem que esses apresentassem problemas bucais. Leão e Dias $(2001)^{7}$ concluíram em seus estudos que o objetivo da faculdade deve ser reforçar a promoção de saúde e prevenção, reconhecer a importância do cuidado clínico, reconhecendo os direitos, responsabilidades e escolhas de cada paciente, mostrando que a esta instituição avaliada deve voltar sua atenção para a prevenção.

Comparando-se os nossos resultados a achados na literatura, os resultados foram semelhantes já que Garcia e Contreras (2002) ${ }^{5}$ sugeriram que o cirurgião dentista deve passar as informações referentes ao tratamento de maneira direta. E como resultados verificou-se que os pacientes estavam satisfeitos com a conduta apresentada pelos estudantes, já que apresentaram um nível alto de satisfação na questão que avaliava se os estudantes sempre explicam o que vão fazer antes de começar o tratamento.

\section{CONCLUSÃo}

De acordo com os resultados obtidos neste estudo, pode-se concluir que os pacientes estão parcialmente satisfeitos com o atendimento oferecido no Curso de Odontologia da Universidade de Franca. Portanto uma maior atenção deve ser dada na realização de campanhas e debates para evitar que os pacientes apresentem problemas bucais e também organizar um atendimento diferencial aos sábados ou organizar turnos de atendimentos a noite.

\section{ABSTRACT}

Analysis of patient satisfaction toward dental services in the Dental Clinic of the University of Franca

Patient care is an essential component of the academic training of future dentists during their undergraduate studies. For this reason, some colleges offer patients free services, under the supervision of a professor. Many evaluations are made to determine the quality of the services rendered; however, the viewpoint of patients is not considered, albeit of fundamental importance. Considering how important the beneficiary of these services is in the teaching-learning process, the objective of this study was to evaluate the satisfaction of patients toward the dental services rendered by the dental clinics of the University of Franca. This evaluation allows the aspects of the dental services rendered to be analyzed and new methods to be developed to give patients appropriate and quality service. Eighty one patients were interviewed with a questionnaire proposed by Davies and Ware Jr. (1982) and adapted for this study. It was composed of 18 questions that evaluated dental services according to access, availability or convenience, cost, continuity, overall satisfaction, pain control, quality, total access and satisfaction rate. The overall satisfaction toward dental services was $63 \%$. The main complaint was the waiting time for receiving dental services. We can conclude that patients are partially satisfied toward the dental services offered by the dental clinics at the University of Franca.

\section{DESCRITORES}

Service evaluation. Dental schools. Satisfaction. -

\section{REFERÊNCIAS}

1. Bottan ER, Sperb RAL, Telles PS, Uriarte Neto M. Avaliação de serviços odontológicos: a visão dos pacientes. Rev da ABENO. 2006 jul.-dez; 6(2):128-33. 
2. Brosky ME, Keefer OA, Hodges JS, Presun IJ, COOK G.Patient perceptions of professionalism in dentistry. J Dent Educ. 2003 Aug; 67(8): 909-15.

3. Cruz JS, Cota LOM, Paixão HH, Pordeus IA.. A imagem do cirurgião-dentista: um estudo de representação social. Rev Odontol Univ São Paulo. 1997 out/dez; 11(4): 307-313.

4. Davies ARE, Ware Junior JE. Develpment of a dental satisfaction questionnaire for the health insurance experiment: Rand Corporation. 1982.

5. Garcia PPNS, Contreras EFR. Estratégias adotadas por cirurgiões-dentistas para a manutenção do paciente no consutório odontológico. Rev Paul Odontol. 2002 jan/fev; 24(1): 27-30.

6. Lahti S, Tuutti H, Hausen H, Kaariainen R. Opinions of different subgroups of dentists and patients about the ideal dentist and the ideal patient. Community Dent Oral Epidemiol. 1995 Apr; 23(2):89-94.

7. Leão ATT, Dias K. Avaliação dos serviços de saúde prestados por faculdades de odontologia: a visão do usuário. Rev Bras Odont Saúde Coletiva. 2001; 2(1): 40-46.

8. Mascarenhas AK. Patient satisfaction with the comprehensive care model of dental care delivery. J Dent Educ. 2001 Nov; 65(11): 1266-71.

9. Newsome PR, Wright GH. A review of patient satisfaction: 1. Concepts of satisfaction. Br Dent J. 1999 Feb; 186(4): 161-5.

10. Newsome PR, Wright GH. A review of patient satisfaction: 2. Dental patient satisfaction an appraisal of recent literature. $\mathrm{Br}$ Dent J. 1999 Feb; 196(5): 166-70.

11. Oliver RL. Congnitive, affective, and attribute bases of the satisfaction response. Journal of consumer research. 1993 Dec; 20(3): 418-30.

Recebido em 14/10/2010

Aceito em 17/12/2010 